\title{
Nitrogen glaciers flow on Pluto
}

Data from New Horizons mission also reveal a hazy atmosphere that is growing colder and thinner.

\section{Alexandra Witze}

24 July 2015

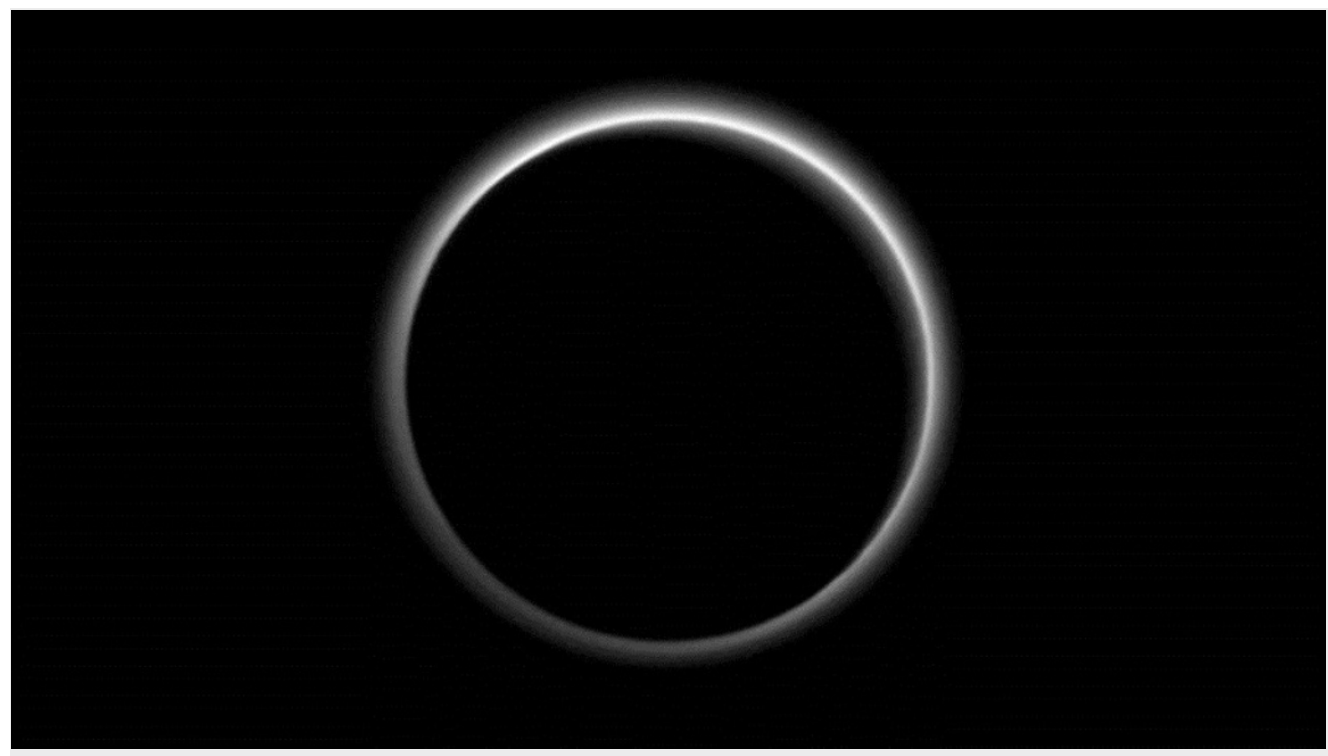

NASA/JHUAPL/SWRI

Pluto's atmosphere glows in this image taken as the dwarf planet passed between the Sun and the New Horizons spacecraft.

Pluto has nitrogen glaciers flowing down from its distinctive, icy heart. And the dwarf planet's thin atmosphere may have begun to freeze out and drift onto its surface as snow - a change expected as Pluto moves farther from the Sun, but never before seen.

Scientists with NASA's New Horizons mission unveiled these findings, and a raft of new images, at a press conference on 24 July, just ten days after their spacecraft flew by Pluto.

During that historic journey, the New Horizons craft measured Pluto's surface pressure for the first time. Scientists then used the reading to estimate the mass of the dwarf planet's atmosphere - and what they found puzzed them.

"The mass of Pluto's atmosphere has decreased by a factor of two in two years," says Michael Summers, a team member and planetary scientist at George Mason University in Fairfax, Virginia. "That's pretty astonishing."

Measurements taken from Earth, starting in the late 1980s when Pluto was closer to the Sun, suggested that Pluto's atmosphere had actually gotten denser in the past couple of decades. This argues against the idea

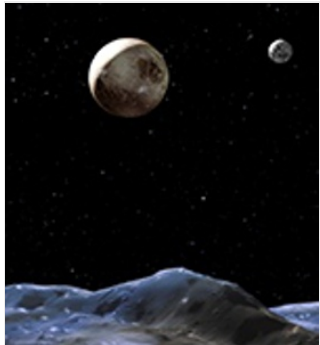

Nature special: Pluto and Ceres that the nitrogen-dominated atmosphere would freeze out and condense on Pluto's surface as the dwarf planet moves away from the Sun.

\section{Strange behaviour}

Summers cautions that the latest observation is just one data point. It comes from a one-off experiment in which radio antennas in NASA's Deep Space Network — located on Earth — beamed a powerful signal to the New Horizons craft as it passed behind Pluto. By measuring how much those radio waves were bent along their journey, the spacecraft was able to measure the surface pressure of Pluto's atmosphere.

The pressure reading is about half of what ground-based astronomers saw in 2013 when they measured Pluto's atmosphere. The New Horizons team is unsure why the atmosphere apparently started to freeze out just before the spacecraft buzzed by the dwarf planet. 
"It would be an amazing coincidence," says the mission's principal investigator, Alan Stern of the Southwest Research Institute in Boulder, Colorado. "But there are some on our team who would say, 'I told you so'."

Another recent experiment could yield some answers. On 29 June, Pluto passed in front — at least to viewers on Earth — of a star, and teams of astronomers were watching. Such an event, known as an 'occultation', can reveal the density of a body's atmosphere. Scientists are currently crunching through the data to see whether they agree with New Horizons' suggestion of a lightweight atmosphere.

"I'm sceptical," says Michael Skrutskie, an astronomer at the University of Virginia in Charlottesville who is helping to lead the analysis. Among other things, he notes, occultation measurements study the atmosphere tens of kilometres above Pluto's surface, whereas the New Horizons' radio measurement was made at its surface. "It's kind of apples and oranges," he says.

\section{Outlook: hazy}

The New Horizons team has also spotted layers of haze in Pluto's remaining atmosphere. These bands extend up to 160 kilometres above the dwarf planet's surface — roughly five times higher than scientists had predicted, Summers says.

Such haze could persist even if much of Pluto's atmosphere has already dissipated, because the particles that make up the fog are tiny and easily lofted.

Pluto's atmosphere comprises gases created when ices sublimate off the dwarf planet's surface — such as the nitrogen, methane and carbon monoxide ices that New Horizons has spotted in the heart-shaped region called Tombaugh Regio.

Close-up images of the edges of nearby fractured plains called Sputnik Planum reveal nitrogen glaciers that reach into a rugged, cratered terrain to the north. At Pluto's frigid temperatures - about $-235^{\circ} \mathrm{C}, 38$ degrees above absolute zero - water ice is too brittle to flow. But nitrogen ice could, if it is roughly a kilometre thick and heated by radioactive decay leaking from Pluto's interior, says William McKinnon, a New Horizons team member and a planetary scientist at Washington University in St. Louis, Missouri.

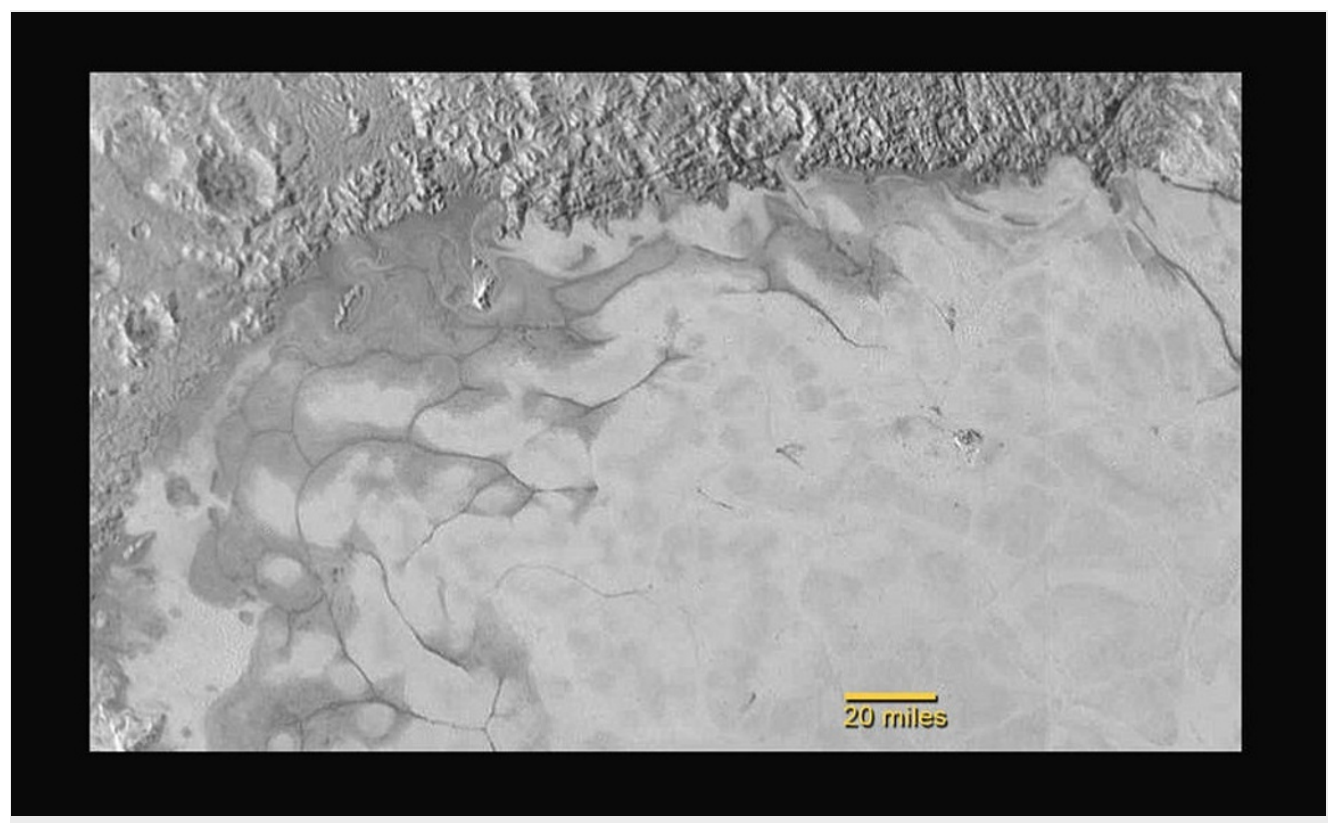

NASAJIHUAPL/SWRI

Patterns of light and dark on Pluto's surface seem to signal flowing nitrogen ice.

\section{Phoning home}

So far, the New Horizons spacecraft has sent back about $5 \%$ of the data that it has collected. On 31 July, it will move into a new mode, in which it begins spinning, which will enable engineers to turn off many of the navigation systems in order to power a second antenna. Using both antennas to transmit the information back to Earth will speed up the data delivery, says Kimberly Ennico-Smith, a planetary scientist at NASA's Ames Research Center in Mountain View, California, and deputy project scientist for the mission. 
wind and space environment.

"The one-shot nature of it has been very exciting," says Carly Howett, a planetary scientist at the Southwest Research Institute. "And just a little bit scary."

Nature | doi:10.1038/nature.2015.18062 